\title{
Perturbation of spermatogenesis by androgen antagonists directly injected into seminiferous tubules of live mice
}

\author{
Kaz Nagaosa ${ }^{1}$, Atsushi Kishimoto ${ }^{1}$, Ryoichi Kizu ${ }^{1}$, Akihisa Nakagawa ${ }^{2}$, Akiko Shiratsuchi ${ }^{1,2}$ \\ and Yoshinobu Nakanishi ${ }^{1,2}$ \\ ${ }^{1}$ Graduate School of Natural Science and Technology and ${ }^{2}$ Graduate School of Medical Science, Kanazawa \\ University, Kanazawa, Ishikawa 920-1192, Japan \\ Correspondence should be addressed to Y Nakanishi; Email: nakanaka@kenroku.kanazawa-u.ac.jp
}

K Nagaosa and A Kishimoto equally contributed to this work

R Kizu is now at Faculty of Pharmaceutical Sciences, Doshisha Women's College of Liberal Arts, Kyotanabe, Kyoto 610-0395, Japan A Nakagawa is now at Department of Molecular, Cellular, and Developmental Biology, University of Colorado, Boulder, Colorado 80309, USA

\begin{abstract}
Natural and artificial substances present in the environment can affect our health. Testicular toxicants in particular are troublesome, because they disturb gonadal function of males. Translocation of substances into the seminiferous epithelium where sperm production proceeds is restricted due to the blood-testis barrier, but this permeability barrier temporarily disappears under physiological and sub-physiological conditions. This means that any substance could enter the seminiferous epithelium and disturb sperm production. To reduce the risk posed by such toxins, it is important to accurately determine which substances possess the toxicity. However, existing assay systems are not satisfactory in terms of both accuracy and sensitivity. Here, we report the establishment of such a system. We injected the androgen antagonists, flutamide and vinclozolin, directly into seminiferous tubules of live mice, which had been treated with busulfan for a temporal arrest of spermatogenesis, and the testes were histologically examined to see the effect of the injected materials on spermatogenesis that was in the process of recovery. The injection of either substance brought about a severe impairment of spermatogenesis at an amount over a million times smaller than that used in the previous assay systems where animals are administered with test substances outside of the testis. In contrast, these androgen antagonists at the same doses showed lesser effects when intratubularly or intraperitoneally administered into mice that had not been pretreated with busulfan. We propose that the method adopted in this study is a novel assay system to identify potential testicular toxicants.
\end{abstract}

Reproduction (2007) 133 21-27

\section{Introduction}

Either natural or artificial substances present in the environment sometimes threaten human health, and such substances include the so-called endocrine disruptors which impair gonadal functions bringing about defects in gamete production. Some of these toxins were recently shown to cause male infertility beyond generations (Anway et al. 2005). In addition, chemical compounds that are synthesized in the process of drug development are often withdrawn due to the discovery of testicular toxicity. Testicular toxicants include chemicals that act as androgen receptor antagonists. The development and the function of androgen-dependent tissues in males are affected on exposure to these chemicals, and some such chemicals cause a decrease in the number of testicular sperm (Gray et al. 2001).

There is a permeability barrier across the epithelium and endothelium of seminiferous tubules where spermatogenic differentiation proceeds. The inter-Sertoli tight junctions constitute this barrier, which is called the blood-testis barrier or the seminiferous epithelium barrier (Lui et al. 2003). The blood-testis barrier protects the seminiferous epithelium from invasion by molecules or cells that may perturb the process of spermatogenesis. At the same time, this permeability barrier needs to be temporarily lost at particular stages of spermatogenesis for the movement of germ cells across the seminiferous epithelium (Lui et al. 2003, Lui \& Lee 2006). In addition, some chemicals disrupt this barrier and increase its 
permeability (Xia et al. 2005). It is presumed that such chemicals force the regulatory mechanism, which controls the opening and closing of the tight junctions under physiological conditions (Lui et al. 2003, Lui \& Lee 2006), to malfunction. Furthermore, tight junctions become structurally and functionally deficient upon infection with some pathogenic microbes (Guttman et al. 2006). This evokes the possibility that chemicals, which normally do not traverse the blood-testis barrier, may invade the seminiferous epithelium and disturb spermatogenesis when the barrier physiologically or accidentally opens. It is therefore necessary to have an assay system whereby potential testicular toxicity of any natural and artificial substances can be assessed. However, existing in vivo assay systems are not accurate or sensitive enough for this purpose.

With existing procedures, test materials, which are mostly water-insoluble, are administered via oral gavage or injected into the peritoneal cavity (US Environmental Protection Agency 1998, O'Connor et al. 2002, Kubota et al. 2003). This method has several disadvantages, including: (1) the administered materials influence spermatogenesis only indirectly unless they traverse the blood-testis barrier and enter the seminiferous epithelium, (2) large amounts of materials need to be injected because they become widely dispersed in the body, and (3) portions of the injected materials are left insoluble due to high concentrations. We anticipated that all these shortcomings could be overcome when test materials are directly introduced into the seminiferous epithelium. For this purpose, we decided to adopt a microinjection technique that was developed by Brinster et al. for the transplantation of male germ cells (Brinster \& Zimmermann 1994, Ogawa et al. 1997). We previously investigated the mechanism and role of the phagocytic removal of apoptotic spermatogenic cells by Sertoli cells by microinjecting various materials into seminiferous tubules of live mice and rats (Kawasaki et al. 2002, Maeda et al. 2002, Nakagawa et al. 2004, 2005). In the present study, we took a similar approach to examine the effect of known androgen antagonists on spermatogenesis in live mice. The results indicated that this procedure could be an accurate, sensitive in vivo assay system to test environmental substances for their potential testicular toxicity or endocrine-disrupting action.

\section{Materials and Methods}

\section{Animals}

All experiments involving animals were conducted according to the protocols that had been approved by the Committee on Animal Experimentation of Kanazawa University. Male ddY mice (10-12-week old, weighing 37-40 g; Nihon SLC, Shizuoka, Japan) were used throughout the study. They were housed at $22-25{ }^{\circ} \mathrm{C}$ under a $12 \mathrm{~h}$ light:12 $\mathrm{h}$ darkness cycle with free access to water and food.

\section{Microinjection into seminiferous tubules of live mice}

To temporarily deplete the seminiferous epithelium of spermatogenic cells, mice were intraperitoneally injected with the anticancer drug busulfan (Sigma; $40 \mathrm{mg} / \mathrm{kg}$ weight) as reported previously (Bucci \& Meistrich 1987). Eight weeks after this injection of busulfan, fluids (about $20 \mu \mathrm{l}$ ) containing various reagents were injected into seminiferous tubules from the efferent duct using a glass needle as described previously (Maeda et al. 2002, Nakagawa et al. 2005). The fluid containing flutamide (Sigma) or vinclozolin (Kanto Chemical, Tokyo, Japan) consisted of PBS with $5 \%(\mathrm{v} / \mathrm{v})$ dimethyl sulfoxide (DMSO; Merck). All fluids were supplemented with $0.05 \%(\mathrm{w} / \mathrm{v})$ Trypan blue, a blue color dye, to monitor the distribution of the injected materials in seminiferous tubules; an injection was considered to be successful when more than a half of the testis was stained blue shortly after the injection, without leakage of the fluid into the interstitial compartment. Mice that had not been treated with busulfan also received intratubular or i.p. injections of the same materials. The mice were maintained as described previously until examination of the testes.

\section{Histological examination of testis sections}

Four weeks after the injection of test substances, mice were killed by cervical dislocation, and testes were isolated. The testes were weighed and immersed successively in Bouin's solution, $70 \%(\mathrm{v} / \mathrm{v})$ ethanol, $100 \%$ ethanol, and xylene before they were embedded with paraffin. Testis sections ( $5 \mu \mathrm{m}$ thick) were prepared, stained with Mayer's hematoxylin and eosin, and examined by microscopy to see the progress of spermatogenesis. Types of testicular cells were determined by morphological examination of the sections, based on the size and shape of nuclei (Hess 1990, Russell et al. 1990).

\section{Data processing and statistical analysis}

Two to seven mice with successful intratubular injections for each dosage of the test substances were subjected to the analysis. One hundred tubule crosssections in each of three different sections prepared from one testis were histologically evaluated for the progress of spermatogenesis with busulfan-administered mice. For the analysis of mice not pretreated with busulfan, ten tubule cross-sections at stage VIII of the spermatogenic differentiation were examined for each testis. Statistical analyses were performed using the Fischer's exact test or the Student's $t$-test, and $P$-values of $<0.05$ were considered significant. 


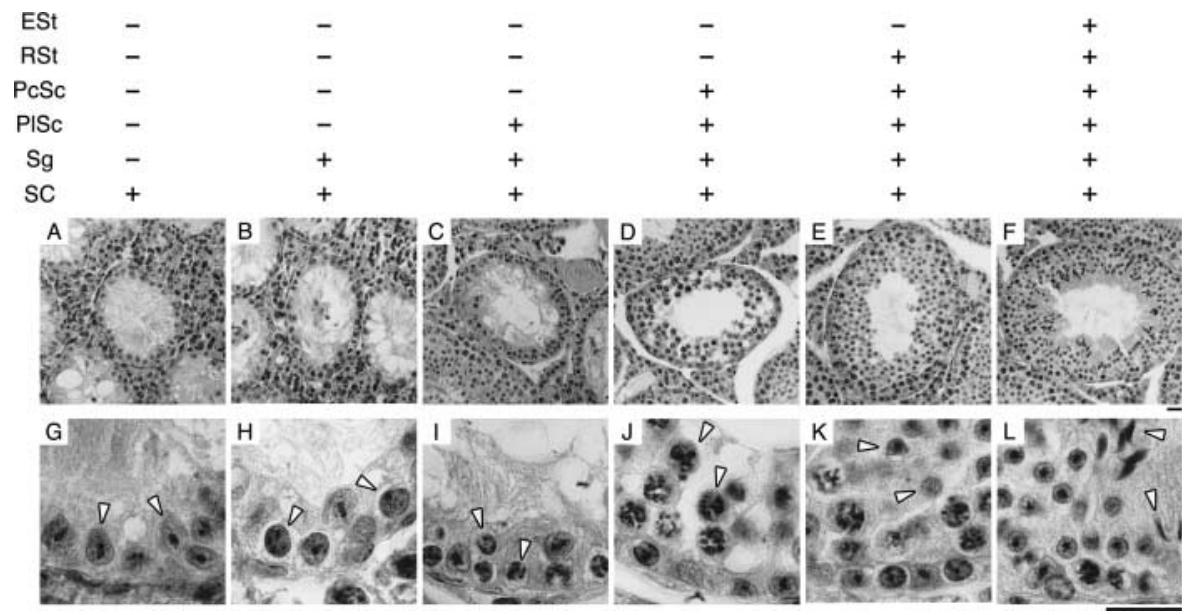

Figure 1 Recovery of spermatogenesis after busulfan-induced arrest. Testes were isolated from mice 12 weeks after the administration of busulfan (with no injection of androgen antagonists), and sections thereof were stained with hematoxylin and eosin to histologically assess the level of spermatogenesis. The lower panels $(\mathrm{G}-\mathrm{L})$ are higher magnified views of the corresponding upper panels $(\mathrm{A}-\mathrm{F})$. Each panel shows an example of a tubule cross-section containing a particular set of testicular cells (presence $(+)$ or absence $(-)$ of each cell type is indicated above the panels). Cells with arrowheads are Sertoli cells (SC) in G, spermatogonia (Sg) in H, preleptotene spermatocytes (PISc) in I, pachytene spermatocytes (PcSc) in J, round spermatids (RSt) in K, and elongated spermatids (ESt) in L. Scale bars represent $20 \mu \mathrm{m}$.

\section{Results}

\section{Temporal arrest and subsequent resumption of sper- matogenesis after treatment with the anticancer drug}

To assess the testicular toxicity of androgen antagonists in mice, we adopted a method whereby drugs are directly injected at the site of spermatogenesis, the seminiferous epithelium (Brinster \& Zimmermann 1994,
Ogawa et al. 1997). In addition, mice were pretreated with the anticancer drug busulfan so that spermatogenesis was temporarily arrested. Spermatogenesis starts to resume in mice about 8 weeks after the administration of busulfan (Maeda et al. 2002, Nakagawa et al. 2005). To see the effects of flutamide and vinclozolin, they were injected into seminiferous tubules at week 8 , and the testes were analyzed for the progress of spermatogenesis
A
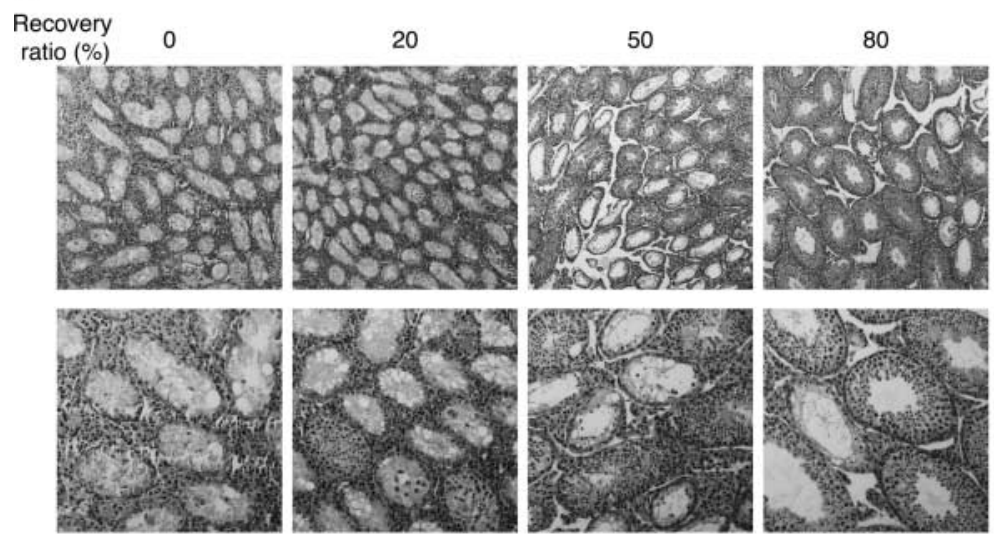

B
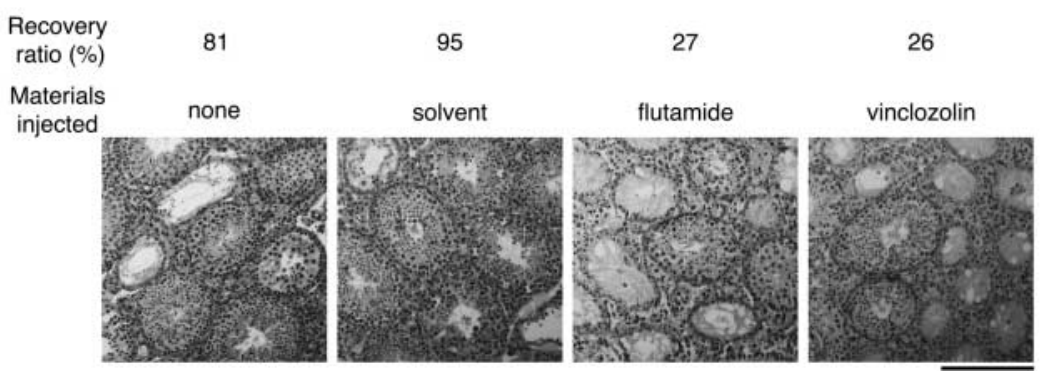

100
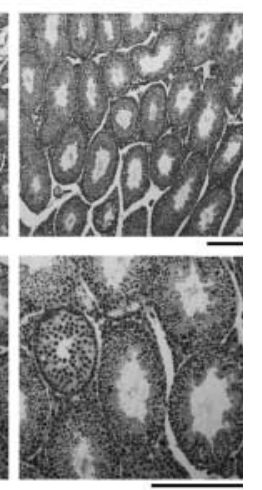

Figure 2 Quantification of recovered spermatogenesis. (A) Examples of testicular sections with the indicated scores for the recovery of spermatogenesis are shown. Spermatogenesis is considered to have recovered when tubule cross-sections contain spermatocytes. The lower panels are higher magnified views of the corresponding upper panels. Scale bars represent $200 \mu \mathrm{m}$. (B) Busulfan-administered mice received intratubular injections of the indicated materials, and examples of testicular sections of the mice are shown with scores for the recovery of spermatogenesis. Scale bar represents $200 \mu \mathrm{m}$. 
after 4 more weeks. We anticipated this procedure to increase the sensitivity of detecting a drug's action, because the injected materials influence spermatogenesis throughout the recovery from the anticancer drugmediated inhibition.

We first established a criterion for the resumption of arrested spermatogenesis. For this purpose, testes of mice that had been treated with busulfan for 12 weeks were analyzed for the level of spermatogenesis; test drugs are to be injected at week 8 , and their effects are to be analyzed after 4 weeks. Paraffin sections of the testes were stained with hematoxylin and eosin so that the testicular cell types were histologically determined. When spermatogenesis does not resume, only Sertoli cells and a small number of spermatogonia, which have survived in the presence of busulfan, should exist in cross-sections of seminiferous tubules. Tubule crosssections with apparently different levels of spermatogenesis were observed even within a single testis (Fig. 1). Most cross-sections contained various types of spermatogenic cells in addition to Sertoli cells as an indication of the proper recovery of spermatogenesis, but some sections showed cells only at early stages of differentiation or were totally devoid of spermatogenic cells. It is likely that the resumption of spermatogenesis was delayed in the latter cross-sections for unknown reasons. Spermatogenesis was considered to have resumed in a tubule cross-section when it contained spermatocytes, and each cross-section was examined for the occurrence of spermatogenesis based on this criterion. The ratio of cross-sections with ongoing spermatogenesis was expressed to evaluate the level of recovery for each testicular section (examples of testes with different scores are shown in Fig. 2A), and representative results with mice that had been intratubularly injected with androgen antagonists are shown in Fig. 2B (see below for details).

\section{Inhibition of spermatogenesis by androgen antagonists during recovery from busulfan-induced arrest}

We chose flutamide (Cook et al. 1993) and vinclozolin (Kelce et al. 1994), which are respectively used as an anticancer drug and a fungicide, as androgen antagonists to be examined for an effect on spermatogenesis by this method. We first examined if the solvent alone disturbs spermatogenesis. Busulfan-treated mice were injected with PBS containing 5\% DMSO (the solvent) or PBS alone, and the level of spermatogenesis in testes of these mice was compared with that in testes of mice that were left uninjected (negative control). We found that spermatogenesis did not resume in about half of the testes even in the control mice that had received no injection, and the injection of the solvent or PBS did not seem to influence the recovering spermatogenesis (Fig. 3A). We then tested the effect of the two androgen antagonists. Varying doses of the antagonists were

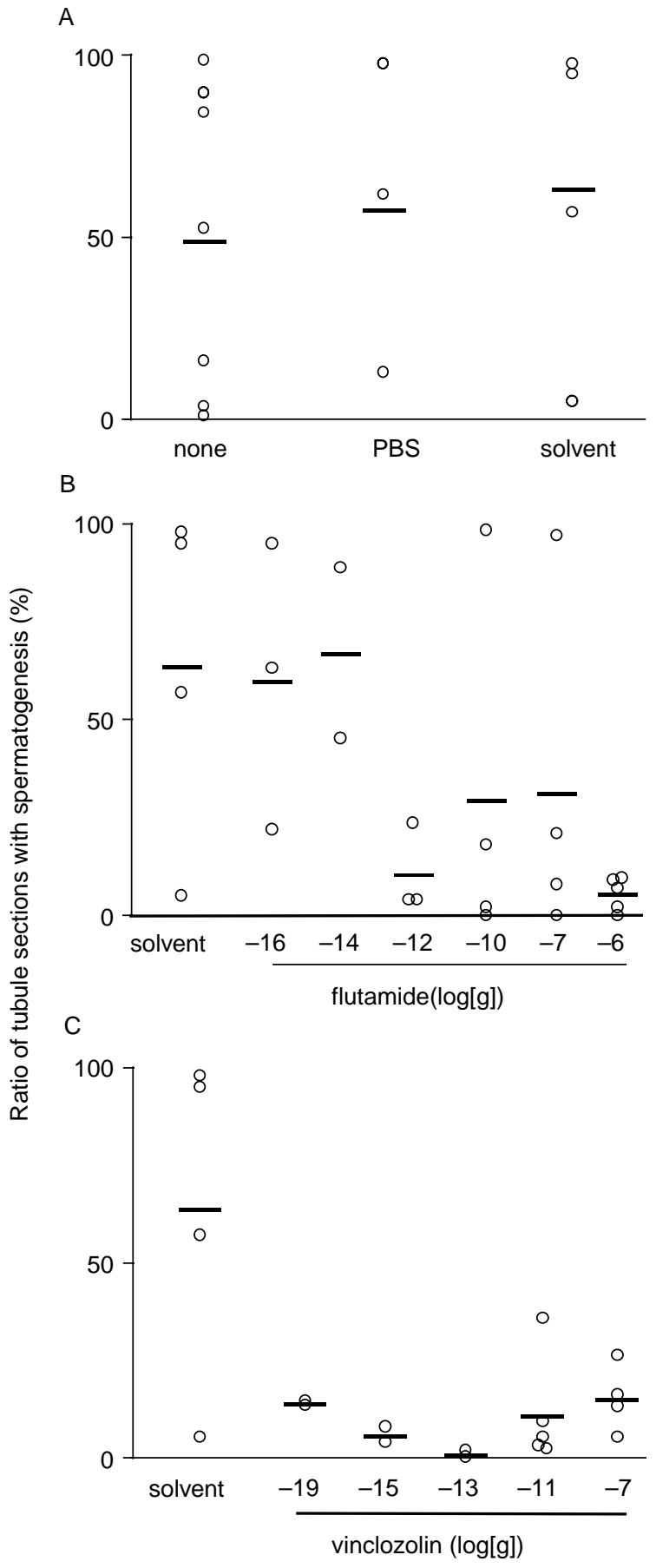

Figure 3 Effect of androgen antagonists on spermatogenesis in busulfanadministered mice. Mice that had been administered with busulfan were microinjected with the indicated materials at seminiferous tubules, and their testes were histologically examined for the level of spermatogenesis. Ratios of tubule cross-sections with recovered spermatogenesis are shown. The horizontal bars in each panel indicate average scores. Each point corresponds to an individual mouse examined.

injected into seminiferous tubules of mice that had been administered with busulfan, and the level of recovering spermatogenesis was determined 4 weeks after the microinjection. At the initial stage of this study, 
we found that one testis of a pair, which had received no injection, often showed a level of spermatogenesis similar to that observed in the testis that had been injected with a test material. This suggests that materials injected into one testis of a mouse influence spermatogenesis occurring in the other testis. This might occur either via a bloodstream-mediated movement of the material to the other testis or through an indirect effect of the material; i.e., perturbation of the secretion of hormones that control spermatogenesis. Whatever the reason, we decided not to inject different materials into a pair of testes. Having a deviation of near $50 \%$, the effect of the androgen antagonists on spermatogenesis could not be assessed on an all-or-none basis. However, either substance showed an inhibitory effect at extremely low doses; $10^{-3}$ ng flutamide inhibited spermatogenesis (Fig. 3B), and vinclozolin caused inhibition at a dose as low as $10^{-10} \mathrm{ng}$ (Fig. 3C). In order to numerically evaluate the results, the number of testes that contained $50 \%$ or more tubule cross-sections with evidence of recovering spermatogenesis was determined and processed. We found that inhibitory effects of both androgen antagonists were statistically significant (Table 1). In previous studies where animals were administered with the materials via oral gavage, effective doses of the materials were reportedly several tens to hundreds of milligrams per kilogram body weight a day for flutamide and vinclozolin (Gray et al. 2001, O'Connor et al. 2002, Kubota et al. 2003), which are well over a million times higher than those observed in this study. When the level of recovered spermatogenesis was compared with body weight, no significant correlation was observed (Fig. 4A). This suggests that the effect of the substances is not strong enough to cause systemic impairment in mice. In contrast, a positive correlation was found between the level of spermatogenesis and testis weight (Fig. 4B). This indicates that the level of spermatogenesis, and therefore the testicular toxicity of the test materials, is predictable by weighing testes instead of histologically examining them.

We next examined if the inhibitory effects of the androgen antagonists were restricted to the particular experimental condition using busulfan-administered mice. For this purpose, the same substances were injected into seminiferous tubules of mice that had not

Table 1 Effect of androgen antagonists on spermatogenesis.

\begin{tabular}{lc}
\hline Materials injected & $\begin{array}{c}\text { Ratio of recovering } \\
\text { testes }(\%)^{\mathrm{a}}\end{array}$ \\
\hline $\begin{array}{lc}\text { Control (none+PBS+ solvent) } \\
\text { Flutamide (doses of } 1 \times 10^{-12} \text { g or higher) }\end{array}$ & $65(9 / 14)^{\mathrm{b}}$ \\
Vinclozolin (all doses) & $13^{\mathrm{c}}(2 / 16)$ \\
\hline
\end{tabular}

${ }^{a}$ Testes containing $50 \%$ or more tubule cross-sections with spermato-

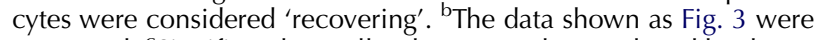
processed. 'Significantly smaller than control as analyzed by the Fischer's exact test: $P<0.01$.

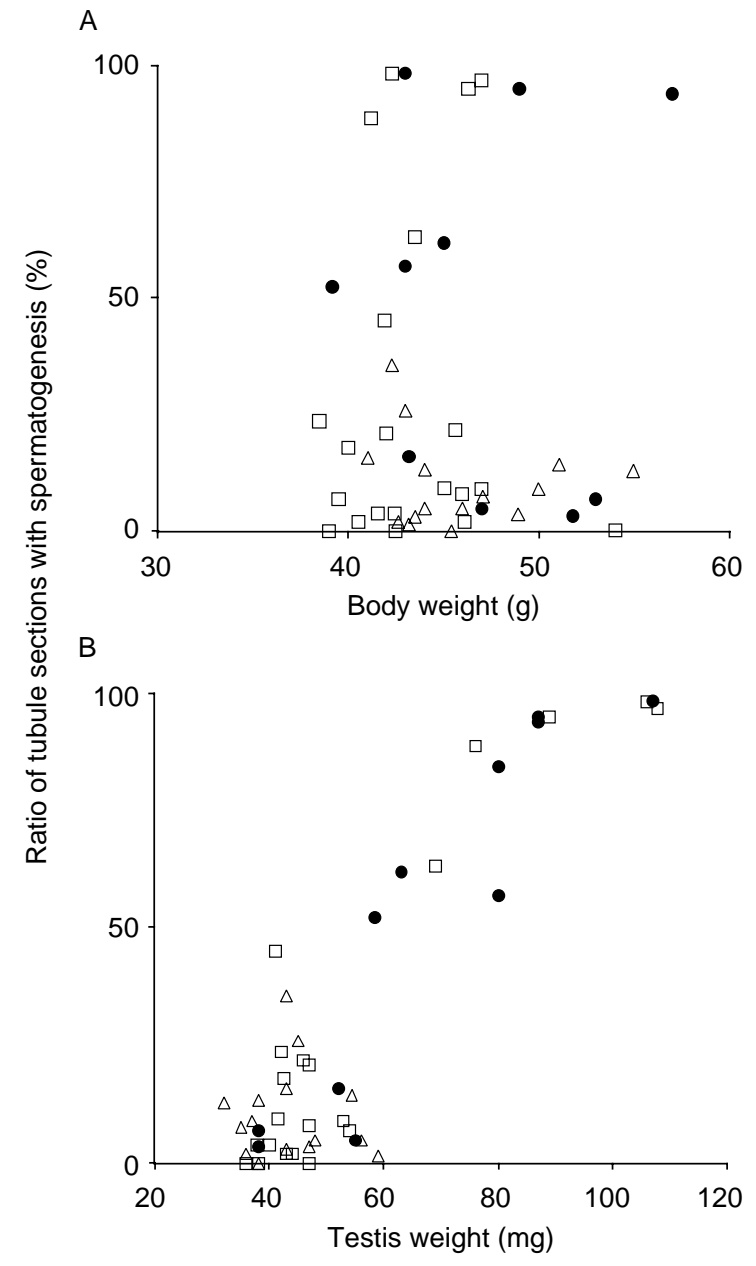

Figure 4 Relation between levels of spermatogenesis and weights of bodies and testes. Ratios of tubule cross-sections with recovered spermatogenesis are shown with the weight of bodies (A) or testes (B) for mice injected with no materials (closed circles), flutamide (squares), or vinclozolin (triangles).

been pretreated with busulfan, and their testes were histologically analyzed. We found that the injection with either antagonist did not cause severe effects; the appearance of tubule cross-sections devoid of spermatogenic cells was not evident (data not shown). However, more precise examination for the progress of spermatogenesis made inhibitory effects of the antagonists apparent. We found that the number of testes containing tubule cross-sections with five or less preleptotene/leptotene spermatocytes per Sertoli cell, of which spermatogenesis we considered to be 'impaired', significantly increased upon injection of either antagonist (Fig. 5A and B). In contrast, i.p. administration of the same substances did not cause the impairment of spermatogenesis (Fig. 5B). These results indicated that the inhibitory effects of intratubularly injected flutamide or vinclozolin are not restricted to busulfan-treated mice, and that to use mice whose spermatogenesis has been 

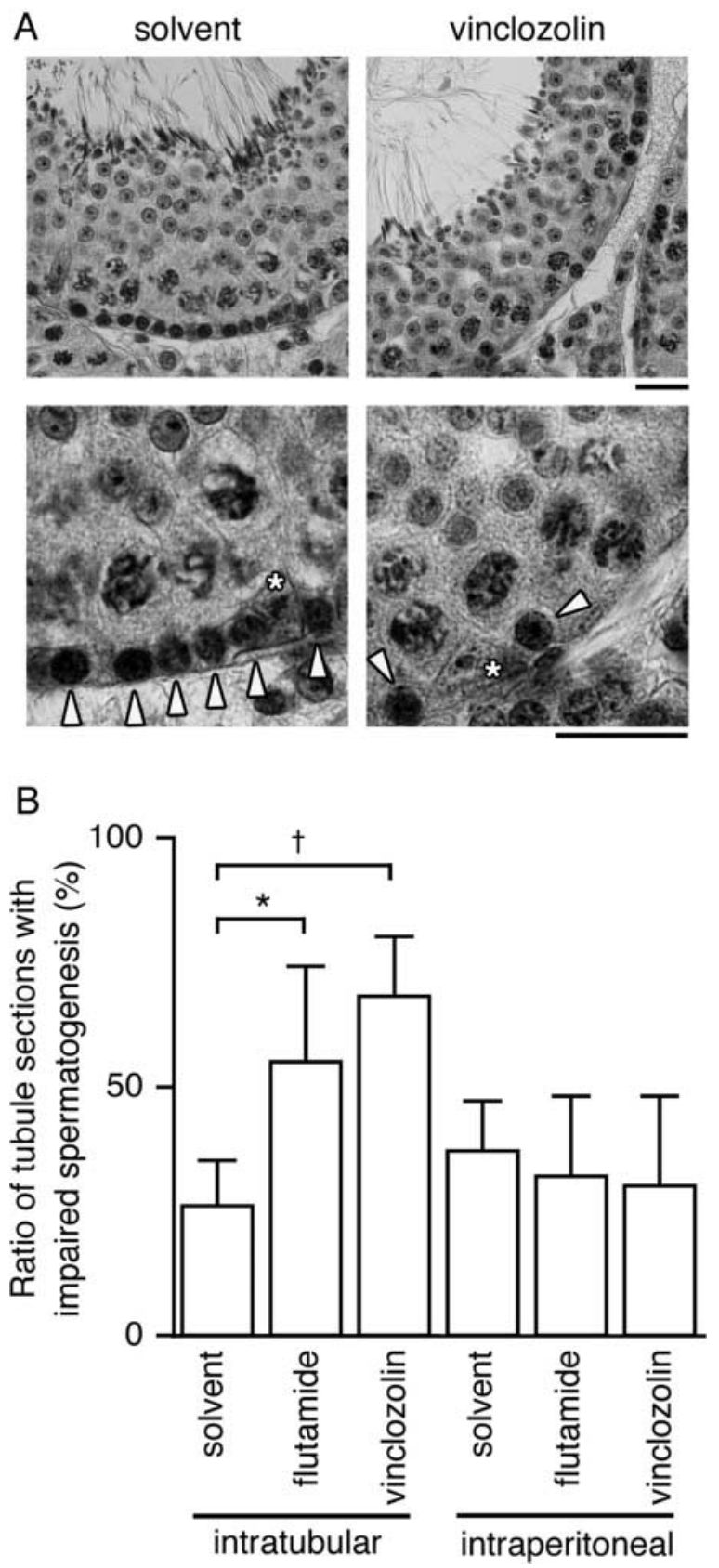

Figure 5 Effect of androgen antagonists on spermatogenesis in mice not administered with busulfan. (A) Testicular sections of mice that have been intratubularly injected with vinclozolin $\left(1 \times 10^{-7}\right.$ g) or solvent alone are shown. The lower panels are higher magnified views of the corresponding upper panels. In the lower panels, Sertoli cells and preleptotene/leptotene spermatocytes are indicated with the asterisks and arrowheads respectively. Scale bars represent $20 \mu \mathrm{m}$. (B) Mice were either intratubularly or intraperitoneally injected with flutamide $\left(1 \times 10^{-6} \mathrm{~g}\right)$, vinclozolin $\left(1 \times 10^{-7} \mathrm{~g}\right)$, or solvent alone, and their testes were histologically examined for the ratio of tubule cross-sections with five or less preleptotene/leptotene spermatocytes per Sertoli cell, 'impaired' spermatogenesis. Significance was analyzed by the Student's $t$-test: ${ }^{*} P<0.02 ;{ }^{\dagger} P<0.0001$. arrested by the treatment with busulfan gives more sensitivity in assessing the effect of injected materials.

\section{Discussion}

In order to develop a sensitive in vivo assay system with which to examine environmental substances for their action as testicular toxicants, we adopted the microinjection of materials into seminiferous tubules of live mice. We maintain that the method used in this study overcomes all shortcomings of existing procedures. When the anticancer drug flutamide or the fungicide vinclozolin was directly introduced into seminiferous tubules of live mice by microinjection, it had an inhibitory effect on spermatogenesis that would otherwise resume after a busulfan-mediated temporal cessation. These chemicals have been known to act as androgen receptor antagonists (Cook et al. 1993, Kelce et al. 1994). It is therefore likely that they have entered Sertoli cells and disrupted the action of androgen. However, the situation could be more complicated, because we observed that substances injected into one testis of a pair often showed the same effect in the other testis of the same mouse. The possibility cannot be excluded that the injected materials entered the bloodstream and reached the other testis or that they disturbed the neural control of hormone secretion necessary for the progress of spermatogenesis. Whatever the mode of action of the drugs, the method described here should be useful for examining environmental substances for testicular cytotoxicity. It seemed to be essential to use mice whose spermatogenesis has been temporarily arrested, because the same chemicals showed only a small effect on spermatogenesis when injected into seminiferous tubules of mice that had not been administered with busulfan. Either androgen antagonist tested impaired the resumption of spermatogenesis at doses in the sub-nanogram range. This means that our method is over million times more sensitive than the methods presently employed. Direct injection of a small volume of fluid is likely to be advantageous in reducing the amount of materials to be tested. Therefore, this procedure may be useful for testing any possible testicular toxicity of substances that exist in small amounts in the environment.

Furthermore, microinjection of materials into seminiferous tubules may help to address other issues associated with spermatogenesis, including the immune response against invading microbial pathogens and the role of metabolites specifically produced there, such as lipids and amino acids. In fact, we have successfully introduced cells into the seminiferous epithelium and clarified the mechanism and roles of the phagocytic elimination of apoptotic spermatogenic cells by Sertoli cells (Maeda et al. 2002, Nakagawa et al. 2005). We hope that this procedure becomes widely used to solve fundamental, applicable issues and problems. 


\section{Acknowledgements}

This study was supported by the Grant-in-Aid for Scientific Study from the Japan Society for the Promotion of Science (no. 70401891) and in part by institutional research grants from Kanazawa University. The authors declare that there is no conflict of interest that would prejudice the impartiality of this scientific work.

\section{References}

Anway MD, Cupp AS, Uzumcu M \& Skinner MK 2005 Epigenetic transgenerational actions of endocrine disruptors and male infertility. Science 308 1466-1469.

Brinster RL \& Zimmermann JW 1994 Spermatogenesis following male germ-cell transplantation. PNAS 91 11298-11302.

Bucci LR \& Meistrich ML 1987 Effects of busulfan on murine spermatogenesis: cytotoxicity, sterility, sperm abnormalities, and dominant lethal mutations. Mutation Research 176 259-268.

Cook JC, Mullin LS, Frame SR \& Biegel LB 1993 Investigation of a mechanism for Leydig cell tumorigenesis by linuron in rats. Toxicology and Applied Pharmacology 119 195-204.

Gray LE Jr, Ostby J, Furr J, Wolf CJ, Lambright C, Parks L, Veeramachaneni DN, Wilson V, Price M, Hotchkiss A, Orlando E \& Guillette L 2001 Effects of environmental antiandrogens on reproductive development in experimental animals. Human Reproduction Update 7 248-264.

Guttman JA, Li Y, Wickham ME, Deng W, Vogl AW \& Finlay BB 2006 Attaching and effacing pathogen-induced tight junction disruption in vivo. Cellular Microbiology 8 634-645.

Hess RA 1990 Quantitative and qualitative characteristics of the stages and transitions in the cycle of the rat seminiferous epithelium: light microscopic observations of perfusion-fixed and plastic-embedded testes. Biology of Reproduction 43 525-542.

Kawasaki Y, Nakagawa A, Nagaosa K, Shiratsuchi A \& Nakanishi Y 2002 Phosphatidylserine binding of class B scavenger receptor type $\mathrm{I}$, a phagocytosis receptor of testicular Sertoli cells. Journal of Biological Chemistry 277 27559-27566.

Kelce WR, Monosson E, Gamcsik MP, Laws SC \& Gray LE 1994 Environmental hormone disruptors: evidence that vinclozolin developmental toxicity is mediated by antiandrogenic metabolites. Toxicology and Applied Pharmacology 126 276-285.

Kubota K, Ohsako S, Kurosawa S, Takeda K, Qing W, Sakaue M, Kawakami T, Ishimura R \& Tohyama C 2003 Effects of vinclozolin administration on sperm production and testosterone biosynthetic pathway in adult male rat. Journal of Reproduction and Development 49 403-412.

Lui W-Y \& Lee WM 2006 Regulation of junction dynamics in the testes - transcriptional and post-transcriptional regulations of cell junction proteins. Molecular and Cellular Endocrinology 250 25-35.

Lui W-Y, Mruk D, Lee WM \& Cheng CY 2003 Sertoli cell tight junction dynamics: their regulation during spermatogenesis. Biology of Reproduction 68 1087-1097.

Maeda Y, Shiratsuchi A, Namiki M \& Nakanishi Y 2002 Inhibition of sperm production in mice by annexin $\mathrm{V}$ microinjected into seminiferous tubules: possible etiology of phagocytic clearance of apoptotic spermatogenic cells and male infertility. Cell Death and Differentiation 9 742-749.

Nakagawa A, Nagaosa K, Hirose T, Tsuda K, Hasegawa K, Shiratsuchi A \& Nakanishi Y 2004 Expression and function of class B scavenger receptor type I on both apical and basolateral sides of the plasma membrane of polarized testicular Sertoli cells of the rat. Development, Growth and Differentiation 46 283-298.

Nakagawa A, Shiratsuchi A, Tsuda K \& Nakanishi Y 2005 In vivo analysis of phagocytosis of apoptotic cells by testicular Sertoli cells. Molecular Reproduction and Development 71 166-177.

O'Connor JC, Frame SR \& Ladics GS 2002 Evaluation of a 15-day screening assay using intact male rats for identifying antiandrogens. Toxicological Sciences 69 92-108.

Ogawa T, Aréchaga JM, Avarbock MR \& Brinster RL 1997 Transplantation of testis germinal cells into mouse seminiferous tubules. International Journal of Developmental Biology $\mathbf{4 1}$ 111-122.

Russell LD, Ettlin RA, Sinha Hikim AP \& Clegg ED 1990 Staging for Laboratory Species, Histological and Histopathological Evaluation of the Testis, pp 62-194.

US Environmental Protection Agency 1998 Endocrine Disruptor Screening and Testing Advisory Committee (EDSTAC) final report.

Xia W, Mruk DD, Lee WM \& Cheng CY 2005 Cytokines and junction restructuring during spermatogenesis - a lesson to learn from the testis. Cytokine and Growth Factor Reviews 16 469-493.

Received 13 June 2006

First decision 14 July 2006

Revised manuscript received 29 September 2006

Accepted 23 October 2006 